\title{
Glycomimetic, Orally Bioavailable LecB Inhibitors Block Biofilm Formation of Pseudomonas aeruginosa
}

\author{
Roman Sommer, ${ }^{\dagger, \S}$ Stefanie Wagner, ${ }^{\dagger, \S}$ Katharina Rox, ${ }^{\S, \|}$ Annabelle Varrot, ${ }^{\#}$ Dirk Hauck, ${ }^{\dagger, \S}$ \\ Eike-Christian Wamhoff, ${ }^{\nabla, \bigcirc}$ Janine Schreiber, ${ }^{\S, \perp}$ Thomas Ryckmans, ${ }^{\diamond}$ Thomas Brunner, ${ }^{\text {II }}$ \\ Christoph Rademacher, ${ }^{\nabla, \bigcirc \odot}$ Rolf W. Hartmann, ${ }^{\ddagger},,++$ Mark Brönstrup, $^{\S, \|}$ Anne Imberty, \\ and Alexander Titz ${ }^{*} \dagger, \S,+\infty$
}

${ }^{\dagger}$ Chemical Biology of Carbohydrates and ${ }^{\ddagger}$ Drug Design and Development, Helmholtz Institute for Pharmaceutical Research Saarland (HIPS), Helmholtz Centre for Infection Research (HZI), D-66123 Saarbrücken, Germany

${ }^{\S}$ Deutsches Zentrum für Infektionsforschung (DZIF), Standort Hannover-Braunschweig, Germany

${ }^{\|}$Chemical Biology and ${ }^{\perp}$ Infection Immunology, Helmholtz Centre for Infection Research, D-38124 Braunschweig, Germany

\#Univ. Grenoble Alpes, CNRS, Centre de Recherche sur les Macromolécules Végétales (CERMAV), 38000 Grenoble, France

${ }^{\nabla}$ Department of Biomolecular Systems, Max Planck Institute of Colloids and Interfaces, D-14424 Potsdam, Germany

ODepartment of Biology, Chemistry and Pharmacy, Freie Universität Berlin, D-14195 Berlin, Germany

- Roche Pharmaceutical Research and Early Development, Roche Innovation Center Basel, CH-4070 Basel, Switzerland

"Biochemical Pharmacology, Department of Biology, University of Konstanz, D-78457 Konstanz, Germany

${ }^{+}$Department of Pharmacy, Saarland University, D-66123 Saarbrücken, Germany

Supporting Information

ABSTRACT: The opportunistic Gram-negative bacterium Pseudomonas aeruginosa is a leading pathogen for infections of immuno-compromised patients and those suffering from cystic fibrosis. Its ability to switch from planktonic life to aggregates, forming the so-called biofilms, is a front-line mechanism of antimicrobial resistance. The bacterial carbohydrate-binding protein $\mathrm{LecB}$ is an integral component and necessary for biofilm formation. Here, we report a new class of drug-like low molecular weight inhibitors of the lectin LecB with nanomolar affinities and excellent receptor binding kinetics and thermodynamics. This class of glycomimetic inhibitors efficiently blocked biofilm formation of $P$. aeruginosa in vitro while the natural monovalent carbohydrate ligands failed. Furthermore, excellent selectivity and pharmacokinetic properties were achieved. Notably, two compounds showed good oral bioavailability, and high compound concentrations in plasma and urine were achieved in vivo.

\section{INTRODUCTION}

The Gram-negative bacterium Pseudomonas aeruginosa has become a severe threat for hospitalized immuno-compromised patients and is now a critical priority 1 pathogen as stated by the WHO in 2017..$^{1-4}$ In persons suffering from cystic fibrosis, chronic infections with this pathogen lead to recurrent pneumonia, sepsis, and lung damage. ${ }^{5}$ Infections of various organs and tissues by this pathogen, such as chronic wound ${ }^{6}$ or catheter-associated urinary tract infections ${ }^{7}$ (caUTI), impose severe clinical challenges which further result from the bacterium's intrinsic drug resistance and additional acquired resistances often leading to multi-drug-resistant or extremely drug-resistant strains (MDR, XDR). ${ }^{8}$ Furthermore, the antimicrobial tolerance of $P$. aeruginosa is enhanced through formation of biofilms, a self-made environment that protects bacteria against host immune defense and antibiotic treat- ment. ${ }^{9,10}$ Triggered by the fact that bacteria inside a biofilm are up to 1000 -fold more resistant toward antibiotics, ${ }^{9}$ the targeting of biofilm formation as therapeutic approach emerged in order to overcome the resistance problem (reviewed in refs 11 and 12).

The two quorum-sensing-regulated ${ }^{13}$ virulence factors LecA $^{14}$ and LecB ${ }^{15}$ (formerly called PA-IL and PA-IIL) are crucial for biofilm formation by $P$. aeruginosa. These two carbohydrate-binding proteins, i.e., lectins, were initially isolated from the clinical isolate PAO1 by Gilboa-Garber et al. ${ }^{16}$ Problematic for therapy, $P$. aeruginosa shows a high genomic diversity among different isolates. ${ }^{17,18}$ While LecA is relatively conserved among strains, the genomic diversity of

Received: October 25, 2017

Published: December 22, 2017 


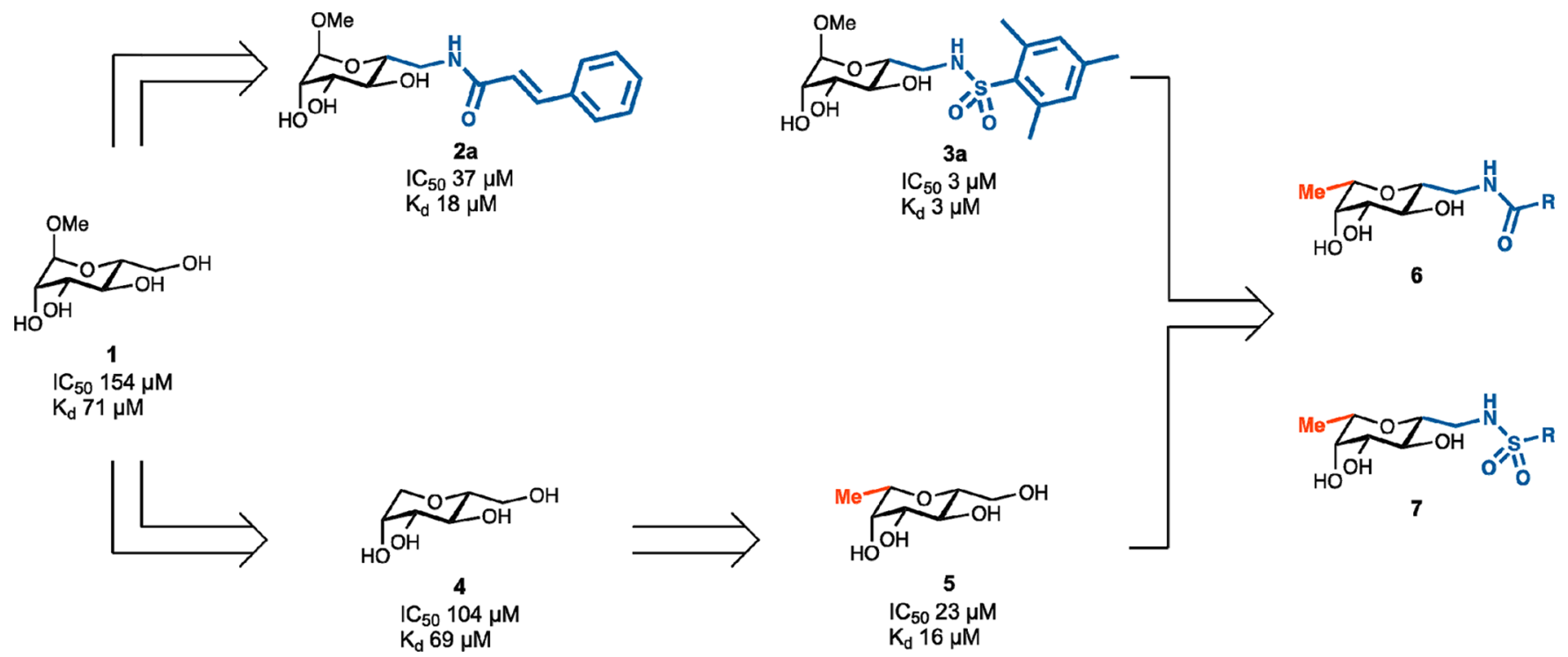

Figure 1. Rational design of $\mathbf{6}$ and 7 as monovalent $C$-glycosidic glycomimetic LecB inhibitors. Derivatives of methyl $\alpha$-D-mannoside $\mathbf{1 - 5}$ and their inhibitory potency for the binding with $\operatorname{LecB}_{\mathrm{PAO1}} \cdot{ }^{30,31,35}$ Moieties colored in blue increase potency by improving binding kinetics. ${ }^{30,32}$ The orange colored methyl group originating from L-fucosides enhances binding to LecB through a lipophilic interaction. ${ }^{31,35}$

LecB varies, and clinical isolates can be grouped into either PAO1- or PA14-like LecB sequence families, with both types covering approximately $50 \%$ each of the clinical isolates. 19,20 However, we could show that despite numerous sequence variations in the two LecB variants, they possess comparable binding specificity which is a prerequisite for targeting a broad range of clinical isolates with one single drug. ${ }^{20}$

The exact mechanism of the lectins' role in biofilm formation is not fully understood. It is assumed, that these tetravalent lectins act as bridging agents to enable glycan-mediated bacterial adhesion to the cellular glycocalix of the host's tissue. In addition, they are believed to mediate contact between bacterial cells through cell-surface glycoconjugates and with the bacterially produced multivalent exopolysaccharides for the establishment of bacterial aggregates and biofilms. ${ }^{11}$ This biofilm-reinforcing property of the lectins, which results from its multivalency, can be blocked by inhibition of the carbohydrate binding sites.

In a mouse infection model, $\mathrm{LecB}$ was shown to be the major determinant for bacterial lung colonization. ${ }^{21,22}$ Furthermore, this protein influences signaling pathways in human lung epithelial cells leading to $\beta$-catenin degradation, a host protein that is crucially involved in tissue repair. ${ }^{23}$ On a structural level, LecB forms noncovalent homotetramers, and each monomer contains two $\mathrm{Ca}^{2+}$-ions, ${ }^{20,24}$ which mediate the binding to its carbohydrate ligands, L-fucose and D-mannose. It has been shown in humans and mice that inhalation of millimolar concentrations of these natural sugars contributed to clearance of bacteria from the lungs and, furthermore, showed synergistic activities with coadministered antibiotics. ${ }^{25,26}$ Due to higher affinities toward fucosides over mannosides, research focused on fucose-based inhibitors with multivalent presentation to further increase avidity. ${ }^{27,28}$ This approach yielded multivalent glycopeptide dendrimer structures with the ability to efficiently inhibit the formation of and disperse established biofilms of $P$. aeruginosa. $^{29}$ Interestingly, in another case of multivalent fucosides, despite nanomolar affinities for LecB, millimolar concentrations $(5 \mathrm{mM})$ have been reported as necessary for efficient inhibition of biofilm formation, and compounds were inactive at $100 \mu \mathrm{M}^{21}$ In the same study, these multivalent inhibitors were even shown to promote bacterial aggregation. Thus, it is conceivable that some multivalent lectin inhibitors can act as mimics of the bacterial exopolysaccharides and therefore unintentionally contribute to biofilm integrity rather than to its desired disintegration.

In order to circumvent such a detrimental biofilm-stabilizing effect of multivalent ligands through cross-linking of the tetravalent lectins inside the biofilm, we have focused on monovalent low molecular weight inhibitors of LecB. ${ }^{30-34}$ In addition, only small molecules can be developed into drug-like molecules with favorable pharmakokinetic properties, i.e., oral application with systemic drug bioavailability, which is necessary to reach all possible types of the various organ and tissue infections elicited by $P$. aeruginosa. Thus, small monovalent molecules hold the premise to circumvent both, biofilm-reinforcing effects and the restriction to topical applications for the multivalent systems.

Here, we report two types of monovalent glycomimetic LecB inhibitors, cinnamides 6 and sulfonamides 7. These new low molecular weight $C$-glycosidic LecB inhibitors showed nanomolar potency against LecB with excellent target selectivity, toxicity, and in vitro $\mathrm{ADME}$ properties. Notably, the glycomimetic sulfonamides developed here were potent inhibitors of biofilm formation in stark contrast to the ineffective native carbohydrates. Finally, a murine in vivo pharmacokinetic study revealed the suitability of these glycomimetics for oral application in a proof-of-concept infection model.

\section{RESULTS AND DISCUSSION}

Design and Synthesis of C-Glycoside LecB Inhibitors. We have previously modified the rather weak LecB ligand methyl $\alpha$-D-mannoside (1) into derivatives with various aromatic substituents attached to $C-6$, which increased potency up to a factor of 20 and addressed a new subsite at the target, e.g., $\mathbf{2 a}-\mathbf{c}$ and $\mathbf{3 a}-\mathbf{c}$, Figures 1 and $2 .{ }^{30,32}$ In contrast to rapidly dissociating $1\left(t_{1 / 2}=45 \mathrm{~s}\right)$, these compounds showed extended receptor residence times with complex half-lives in the 5-20 

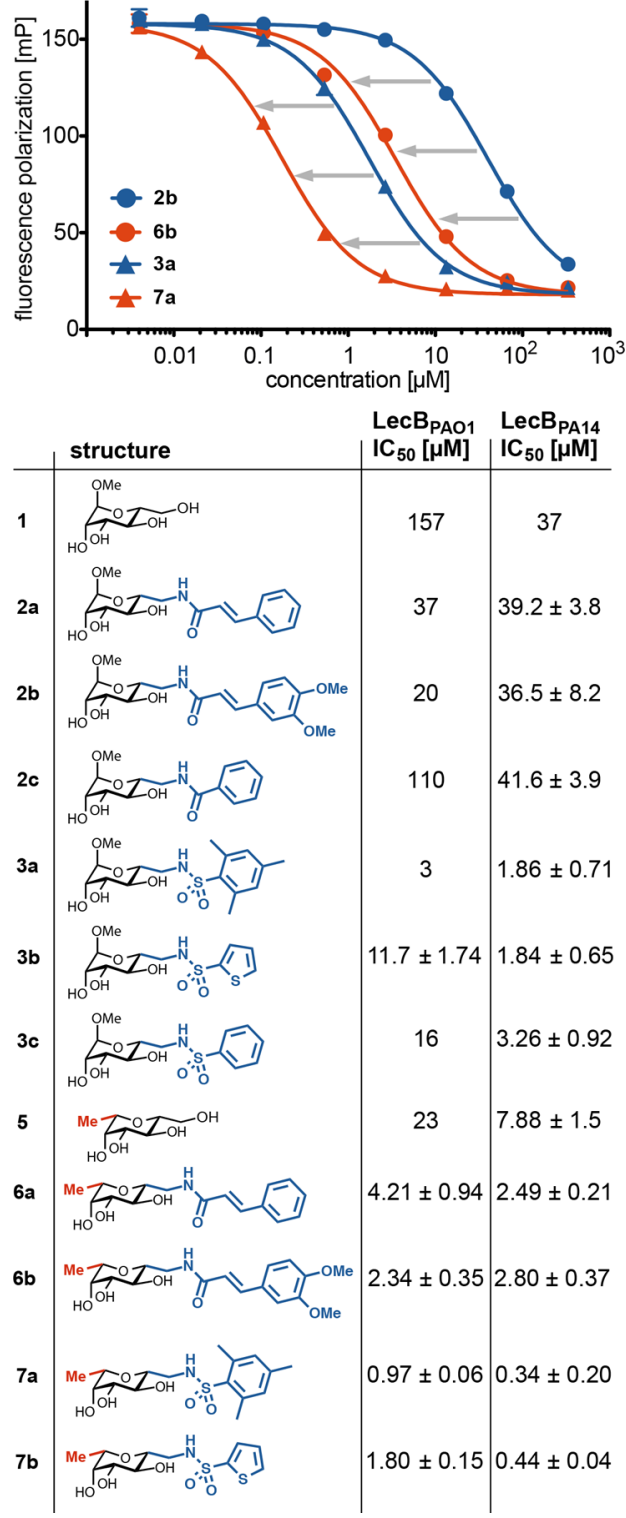

Figure 2. Competitive binding assay of inhibitors with $\mathrm{LecB}_{\mathrm{PAO} 1}$ and $\mathrm{LecB}_{\mathrm{PA} 14}$. Means and standard deviations were determined from a minimum of three independent measurements. $\mathrm{IC}_{50}$ values for $\mathbf{1}$ with both LecB variants and $2 \mathrm{a}-\mathrm{c}, \mathbf{3 a , c}$ and $\mathbf{5}$ with $\mathrm{LecB}_{\mathrm{PAO} 1}$ were previously published. ${ }^{20,30-32}$ One representative titration with $\operatorname{LecB}_{\mathrm{PA} 14}$ is depicted for reference pairs $\mathbf{2 b} / \mathbf{6} \mathbf{b}$ and $3 \mathbf{a} / 7 \mathbf{a}$. Gray arrows indicate the increase in activity for $C$-glycosides. min range, revealing the molecular basis for the increased potency. This property is an important requirement for in vivo efficacy. In order to further optimize target interactions and increase stability toward hydrolases, we merged functional groups establishing attractive interactions with LecB from the natural ligands into one molecule $(\mathbf{1} \rightarrow \mathbf{5}$, Figure 1$) .{ }^{31}$ The equatorial methyl group in 5 resulted in $C$-glycosides with 4 - to 6 -fold enhanced affinities originating from the reported lipophilic contact of the L-fucose methyl group with the lectin. ${ }^{20,24,35}$ Despite these numerous improvements, the previously reported molecules display only moderate affinities for LecB in the range of 3.3-16 $\mu \mathrm{M}$, which is insufficient for application. Now, we aimed to combine the individually optimized structures of mannose-derivatives $2 \mathbf{a} / 3 \mathbf{a}$ and $C$ glycoside 5 into monovalent drug-like molecules 6 and 7 to increase affinity toward the target and assess their biological properties. Furthermore, these new glycomimetic structures, 6 and 7 , are expected to be more stable against host metabolism compared to their glycosidic precursors, resulting from the absence of a labile glycosidic linkage. This design should enable higher compound concentrations in vivo.

To access $\beta$ - $C$-glycosides 6 and 7 , we utilized the previously described synthesis of amine $10,{ }^{31}$ where L-fucose $(8)$ was transformed to $\beta$-C-glycoside 9 by Henry addition of nitromethane under basic conditions followed by a reduction to yield amine 10 (Scheme 1). Then, fucose/mannose hybridtype structures 6 and 7 bearing amide or sulfonamide substituents, respectively, were generated by coupling with different electrophiles.

Besides the amide-bridged cinnamic acid moiety as present in $\mathbf{2 a}$ and $\mathbf{6 a}$ or the sulfonamide-bridged trimethylphenyl moiety in $3 \mathbf{a}$ and $7 \mathbf{a}$, a thiophenesulfonamide was introduced in mannoside $\mathbf{3 b}$ and $C$-glycoside $7 \mathbf{b}$ as a bioisoster of the potent phenylsulfonamide $3 \mathrm{c}^{30}$ in the mannose-series. The final coupling step yielded amides $\mathbf{6} \mathbf{a}, \mathbf{b}$ and sulfonamides $7 \mathbf{a}, \mathbf{b}$ in moderate to good yields (33-72\%, over 2 steps).

Improved Binding Properties of C-Glycoside Structures toward LecB. Derivatives $\mathbf{6} \mathbf{a}, \mathbf{b}$ and $7 \mathbf{a}, \mathbf{b}$ were then analyzed for their capacity to inhibit the two representative lectin variants of the two clinically occurring strain clades, $\mathrm{LecB}_{\mathrm{PAO} 1}$ and $\mathrm{LecB}_{\mathrm{PA1} 4}$, based on previously established competitive binding assays ${ }^{20,30}$ (Figure 2). Furthermore, all

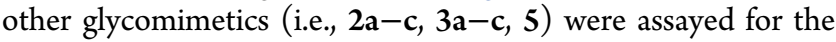
first time also toward $\mathrm{LecB}_{\mathrm{PA} 14}$ and showed stronger binding to the PA14 variant compared to that toward $\mathrm{LecB}_{\mathrm{PAO} 1}$, with the exception of cinnamides $\mathbf{2 a} \mathbf{a} \mathbf{b}$. In general, enhanced affinities were detected for $C$-glycoside derivatives $\mathbf{6 a}, \mathbf{b}$ and $7 \mathbf{a}, \mathbf{b}$ compared to those of their respective mannose analogs $\mathbf{2} \mathbf{a}, \mathbf{b}$

Scheme 1. Synthesis of Structures $6 a, b$ and $7 a, b^{a}$

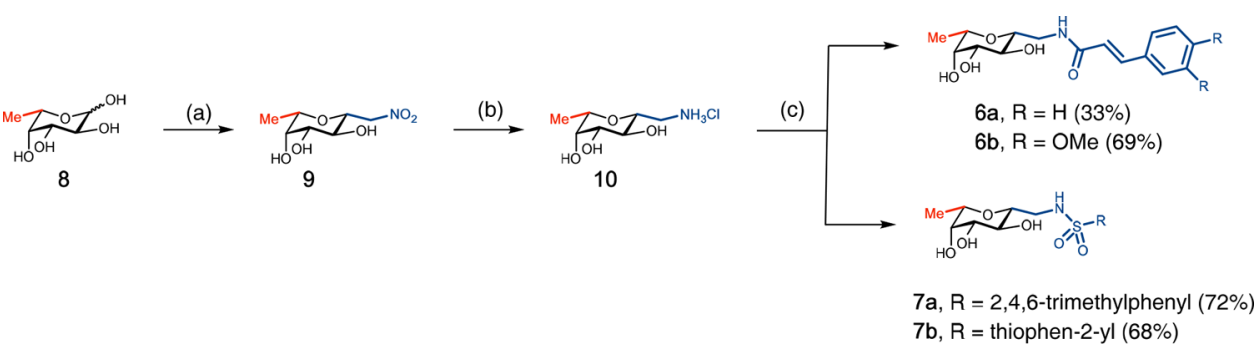

$7 \mathrm{~b}, \mathrm{R}=$ thiophen-2-yl $(68 \%)$

${ }^{a}$ Reagents and conditions: (a) $\mathrm{MeNO}_{2}$, DBU, molecular sieves $3 \AA$, 1,4-dioxane, $50{ }^{\circ} \mathrm{C}, 3$ days; (b) Pt/C, $\mathrm{H}_{2}, \mathrm{HCl}, \mathrm{MeOH}, \mathrm{rtt}, 2$ days; (c) acyl/ sulfonyl chloride or carboxylic acid/EDC. $\mathrm{HCl}, \mathrm{Et}_{3} \mathrm{~N}, \mathrm{DMF}, \mathrm{O}^{\circ} \mathrm{C}$. Yields are given over two steps from nitro derivative 9. 


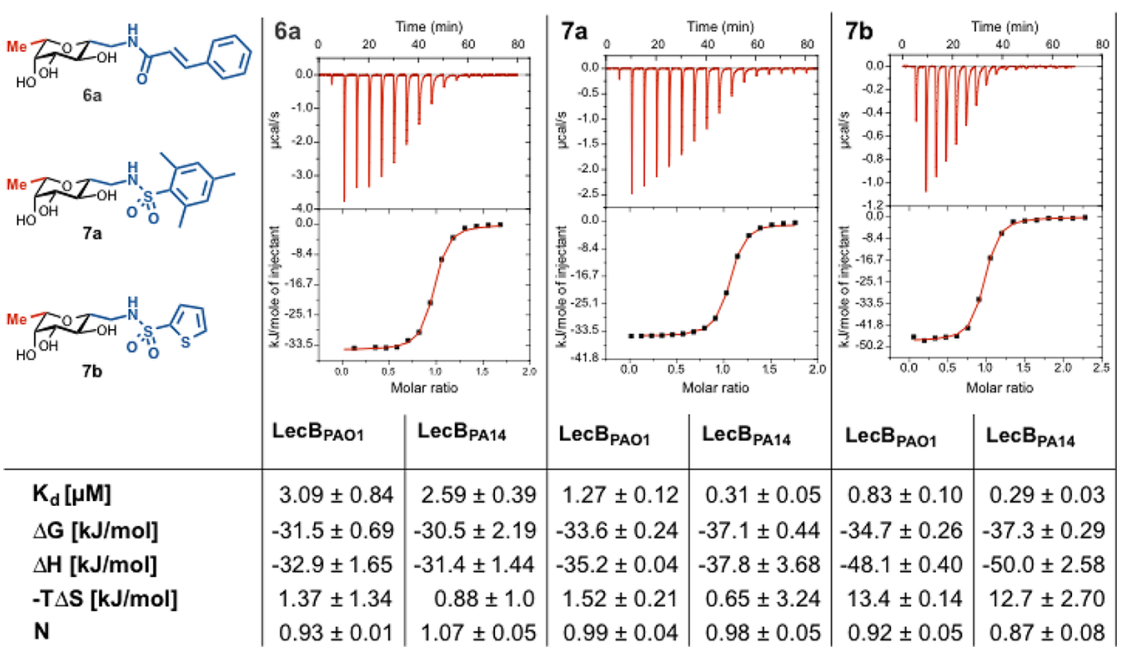

Figure 3. Isothermal titration microcalorimetry of $\mathrm{LecB}_{\mathrm{PAO} 1}$ and $\mathrm{LecB}_{\mathrm{PA} 14}$ with ligands $\mathbf{6 a}$ and $\mathbf{7 a}, \mathbf{b}$. Means and standard deviations were determined from a minimum of three independent titrations. One representative titration graph is depicted for $\mathrm{LecB}_{\mathrm{PAO} 1}$ only.

and 3a,b. Cinnamide 6a $\left(\mathrm{IC}_{50}\left[\operatorname{LecB}_{\mathrm{PAO} 1}\right] 4.21 \mu \mathrm{M}\right.$; $\left.\mathrm{IC}_{50}\left[\mathrm{LecB}_{\mathrm{PA} 14}\right] 2.49 \mu \mathrm{M}\right)$ showed 9- to 16 -fold higher affinities toward both LecB types compared to that of its mannose reference 2a $\left(\mathrm{IC}_{50}\left[\mathrm{LecB}_{\mathrm{PAO} 1}\right] 37 \mu \mathrm{M}^{30} \mathrm{IC}_{50}\left[\mathrm{LecB}_{\mathrm{PA} 14}\right] 39\right.$ $\mu \mathrm{M})$. Sulfonamide $7 \mathrm{a} \quad\left(\mathrm{IC}_{50}\left[\mathrm{LecB}_{\mathrm{PAO} 1}\right] \quad 0.97 \mu \mathrm{M}\right.$; $\left.\mathrm{IC}_{50}\left[\mathrm{LecB}_{\mathrm{PA} 14}\right] 0.34 \mu \mathrm{M}\right)$ was a 3- to 5-fold stronger ligand of LecB compared to that of corresponding mannoside $3 \mathrm{a}$, exceeding the potency of high-affinity natural ligand $\mathrm{L}$-fucose (8) for both lectin types $\left(\mathrm{IC}_{50}\left[\mathrm{LecB}_{\mathrm{PAO} 1}\right] 2.74 \mu \mathrm{M}^{30}\right.$ $\left.\mathrm{IC}_{50}\left[\mathrm{LecB}_{\mathrm{PA} 14}\right] 0.91 \mu \mathrm{M}\right) .{ }^{20}$ On the basis of the four reference pairs analyzed, i.e., mannoside versus $C$-glycoside $2 \mathbf{a} / \mathbf{6 a}, \mathbf{2 b} / \mathbf{6} \mathbf{b}$, $3 \mathbf{a} / 7 \mathbf{a}$, and $3 \mathbf{b} / 7 \mathbf{b}$, which differ only at the anomeric position of mannose, a strong increase in affinity toward LecB of up to 16fold was identified for the $C$-glycosides. In general, the introduction of amide and sulfonamide substituents always improved affinity for LecB compared to that of unsubstituted reference hybrid-structure $5 .^{31}$ Importantly, for the first time the two clinically relevant variants ${ }^{20}$ of LecB were analyzed with glycomimetic inhibitors, which showed potent inhibition for both strain types.

The thermodynamics of binding of derivatives $\mathbf{6} \mathbf{a}$ and $\mathbf{7 a}, \mathbf{b}$ to both LecB variants was then studied by isothermal titration calorimetry (Figure 3, Table S1-3). All ligands showed $K_{\mathrm{d}}$ values in the low micro- to nanomolar range and a 1:1 binding stoichiometry, confirming the $\mathrm{IC}_{50}$ data obtained. Notably, compared to carbocyclic derivatives $6 \mathbf{a}$ and $7 \mathbf{a}(\Delta H=-37.8$ to $-31.4 \mathrm{~kJ} / \mathrm{mol}$ ), thiophene-containing ligand $7 \mathbf{b}$ showed an enhanced enthalpy driven binding $(\Delta H=-50.0$ and $-48.1 \mathrm{~kJ} /$ $\mathrm{mol}$ ), which was partially compensated by disfavored entropic contributions $(-T \Delta S=13.4$ and $12.7 \mathrm{~kJ} / \mathrm{mol})$. Thus, the introduced substituents also significantly enhanced binding enthalpy compared to the unsubstituted congener $5(\Delta H=$ $-27.5 \mathrm{~kJ} / \mathrm{mol}$ ). An increased enthalpic contribution to binding was previously introduced as a benchmark parameter assigned to a higher degree of target selectivity due to a better quality of the drug-target interaction. ${ }^{36}$ Furthermore, physicochemical metrics such as ligand efficiency and related ones, thought to gauge the quality of a drug-target interaction, ${ }^{37}$ are promising. Importantly, the most potent compound thiophene, $7 \mathbf{b}$, displayed enthalphy-driven nanomolar affinities toward both LecB variants.
We have previously detected a strong increase of the receptor half-lives for inhibitors $\mathbf{2} \mathbf{a}, \mathbf{b}$ and $\mathbf{3 a}$, compared to that of to natural carbohydrate ligand methyl $\alpha$-D-mannoside $(\mathbf{1}) .^{32}$ The role of ligand-receptor binding kinetics is becoming increasingly significant for drug discovery and translation of in vitro binding kinetics into cellular or in vivo effects was discussed. ${ }^{38}$ For the in vivo efficacy of a drug molecule, prolonged receptor residence times are of importance. Ligandreceptor binding kinetics of $\mathrm{C}$-glycosides $6 \mathbf{a}$ and $7 \mathbf{a}$ were studied by surface plasmon resonance (SPR) with surfacebound $\mathrm{LecB}_{\mathrm{PAO} 1}$ (Figure 4). With its affinity of $330 \mathrm{nM}$ obtained from SPR, the hybrid-type structure $7 \mathbf{a}$ is a 140 -fold
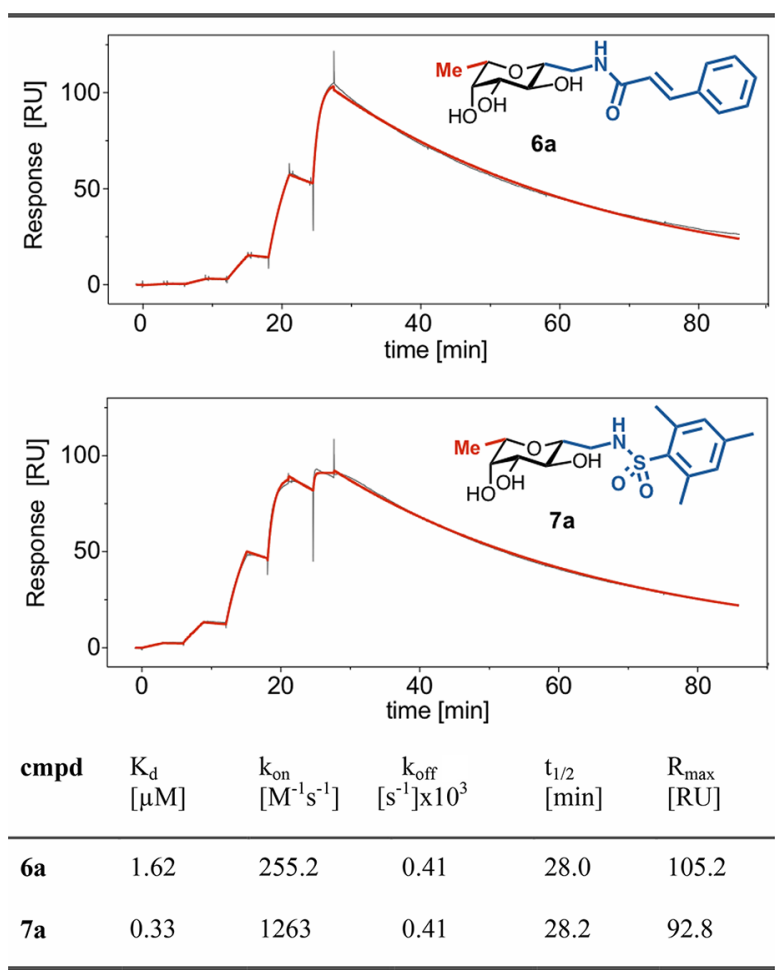

Figure 4. Surface plasmon resonance (SPR) analysis of direct binding of $6 \mathbf{a}$ or $7 \mathbf{a}$ to immobilized $\mathrm{LecB}_{\mathrm{PAO} 1}$. Experimental data in black; calculated fits using a 1:1 binding model in red. 
more potent ligand of LecB than its carbohydrate ligand $\mathbf{1}$ and is 10 -fold more potent than the natural high affinity ligand $\mathrm{L}^{-}$ fucose (8) (see ITC experiments; $K_{\mathrm{d}}$ for $82.9 \mu \mathrm{M}$ ). ${ }^{39}$ Most importantly, a further increase of the drug-receptor half-lives for the $C$-glycosides $\mathbf{6 a}$ and $7 \mathbf{a}$ over those of their mannose-based analogs $2 \mathrm{a}\left(t_{1 / 2} 6.3 \mathrm{~min}\right.$ and $K_{\mathrm{d}}$ by SPR $\left.18.1 \mu \mathrm{M}\right)$ and $3 \mathrm{a}\left(t_{1 / 2}\right.$ $18.6 \mathrm{~min}$ and $K_{\mathrm{d}}$ by SPR $\left.1.12 \mu \mathrm{M}\right)^{32}$ was observed. Receptor residence times of $28 \mathrm{~min}$ for $\mathbf{6 a}$ and $7 \mathrm{a}$ resulted from very slow off rates of $4.1 \times 10^{-4} \mathrm{~s}^{-1}$ of these $C$-glycosides. Thus, these glycomimetics form a 38 -fold more stable complex with the protein, compared to the natural ligand methyl $\alpha$-D-mannoside $\left(1, t_{1 / 2} 0.75 \mathrm{~min}, K_{\mathrm{d}}\right.$ by SPR $\left.47.5 \mu \mathrm{M}\right) .^{32}$ These data validated the results from the competitive binding assay and ITC and further stress the impact of the additional equatorial methyl group in our glycomimetics.

Structure of LecB in Complex with Hybrid Inhibitors. To study the binding mode of the hybrid-type LecB inhibitors, we performed crystallization of these ligands in complex with the lectin. Crystals of two ligands $7 \mathbf{a}$ and $7 \mathbf{b}$ in complex with Lec $B_{\text {PA14 }}$ were obtained by the hanging drop cocrystallization method and the structures were solved to $1.65 \AA$ resolution (Figure 5, Table S4). In both complexes, the carbohydratebinding sites were occupied with the respective inhibitors except one site in the LecB/7a complex.

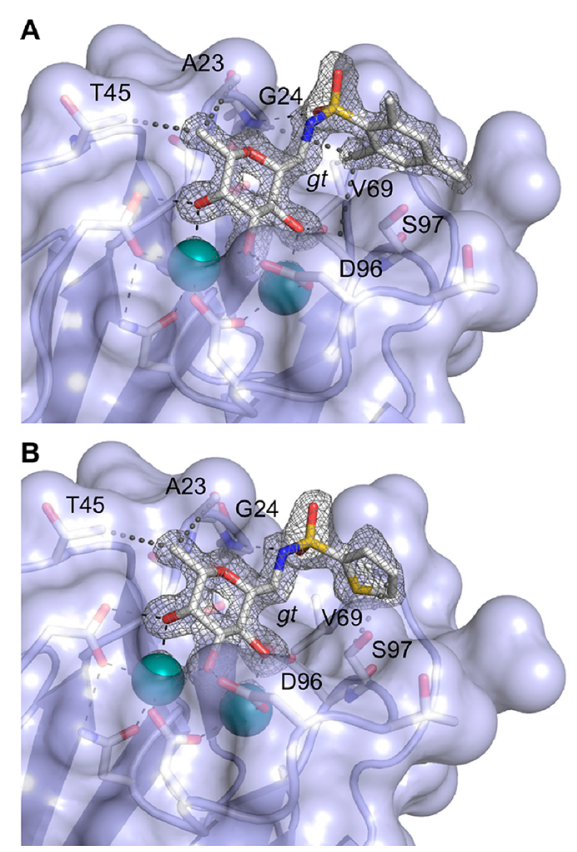

Figure 5. Crystal structures of $\mathrm{LecB}_{\mathrm{PA} 14}$ with $C$-glycoside ligands: (A) Complex with trimethylphenyl sulfonamide $7 \mathbf{a}(1.65 \AA$ resolution), (B) Complex with thiophene $7 \mathbf{b}$ (1.65 Å resolution); For the ligands the $2 F_{\text {obs }}-F_{\text {calc }}$ electron density is displayed at $1 \sigma$. Ligands and amino acids of the carbohydrate recognition domain (CRD) are depicted as sticks colored by elements (C: gray, N: blue, O: red, S: yellow); protein surface in transparent blue and two $\mathrm{Ca}^{2+}$-ions in the binding sites are shown as green spheres.

Surprisingly, a slightly different binding mode for methyl containing analog $7 \mathbf{a}$ was observed in contrast to the previously reported structure ${ }^{30}$ of its mannose analog 3a (Figures 5A, S1, and S3). While the carbohydrate-derived ring is in an identical position, the aromatic moiety attached to the exocyclic $\mathrm{CH}_{2}$ is rotated and located in a cleft of the protein surface that is enclosed by two neighboring loops (S68-D75 and E95-D104) of the lectin. The methyl group attached to the aromatic ring interacts with hydrophobic patches formed by V69 and a $\mathrm{CH}_{2}$ group of D96; one sulfonamide oxygen of the ligand establishes a hydrogen bond with the backbone amide of G24. In contrast with mannoside $3 \mathrm{a}$, a lipophilic contact between the protein surface (G24, V69) and the mesitylene-ring was observed together with a hydrogen bond of its sulfonamide nitrogen with the side chain of D96 (Figure S1). The latter interaction results from a conformational change of the $\mathrm{C} 5-\mathrm{C} 6$ bond in the carbohydrate from a gt-orientation ( $\mathrm{N}$ gauche to $\mathrm{O} 5$ and trans to O4), as present in the complex of LecB with D-mannose (PDB code for LecB $\mathrm{PAO}_{1} 1 \mathrm{OUR}^{40}$ or $\left.\mathrm{LecB}_{\mathrm{PA} 14} 5 \mathrm{~A} 6 \mathrm{Y}\right),{ }^{20}$ to a tgconformation in the mannoside $3 \mathrm{a}$ enabling this observed $\mathrm{NH}$ D96 hydrogen bond. A binding mode comparable to that in $C$ glycoside $7 \mathbf{a}$ was detected for its analog thiophene $7 \mathbf{b}$ (Figures $5 \mathrm{~B}, \mathrm{~S} 1$, and S3). In the structure of mannoside $3 \mathrm{a} / \mathrm{LecB}$, a crystal-lattice-dependent $\pi$-stacking of the aromatic moiety with a second ligand from a neighboring LecB tetramer is observed with all ligands in this structure showing the tg-conformation (Figure S2). Therefore, we consider the binding modes reported here with the gt-orientation displayed by $C$-glycosides $7 \mathbf{a}$ and $7 \mathbf{b}$ as the relevant ones, where crystal induced contacts are absent. In addition to the sulfonamide substituent interactions, a lipophilic contact of the introduced C-glycosidic methyl group with the protein residues T45 and A23 is observed in both analyzed complexes of hybrid-type ligands providing a molecular basis for the measured affinity enhancement.

Low Molecular Weight Glycomimetics Are Potent Inhibitors of Bacterial Biofilm Formation. We then tested the most promising compounds in a $P$. aeruginosa biofilm assay. Most published biofilm assays require staining and washing steps which impact the reproducibility in our hands. Therefore, we generated genetically modified $P$. aeruginosa which constitutively and intracellulary expresses the red-fluorescent protein mCherry from the pMP7605 plasmid. $^{41}$ Because fluorescence intensity directly correlated with cell density (Figure S4), mCherry-derived fluorescence was exploited as an internal stain for bacterial visualization and quantification of biofilm formation by confocal laser scanning fluorescence microscopy. By eliminating washing and staining steps, this method improved reproducibility through in situ imaging. A set of selected compounds comprising natural carbohydrates (mannoside 1, fucoside 11), glycomimetic mannose-derivatives (2a, 3a), and C-glycosides (6a, 7a,b) was selected for assessment of their potential to inhibit biofilm formation by $P$. aeruginosa.

To exclude any unwanted antibiotic effect on biofilm formation, the total fluorescence intensity was recorded after bacterial growth in the presence of compounds for $23 \mathrm{~h}$. Bacteria grew to the same density as the DMSO control under each condition tested (Figure S5). Thus, these results clearly indicated the absence of bactericidal or bacteriostatic effects for the tested compounds.

For inhibition of biofilm formation, culture medium was inoculated with $P$. aeruginosa PA14/mCherry in the presence of $100 \mu \mathrm{M}$ compounds, and culture and biofilm growth was allowed for $48 \mathrm{~h}$, when biofilm mass was quantified using a confocal fluorescence microscope (Figure 6). All analyzed compounds reduced biofilm formation by $P$. aeruginosa. Remarkably, the natural carbohydrate ligands methyl $\alpha$-Dmannoside (1) and methyl $\alpha$-L-fucoside (11) showed only a small reduction in biofilm mass which was statistically 


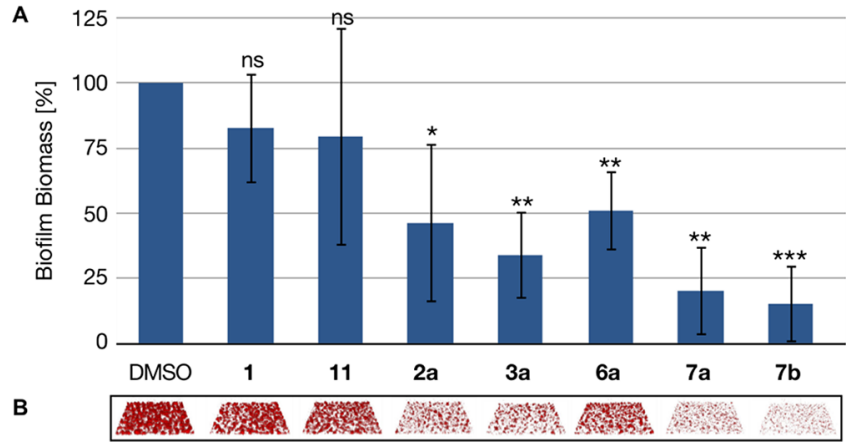

Figure 6. Inhibition of biofilm formation by $P$. aeruginosa after $48 \mathrm{~h}$ growth in the presence of compounds $1,2 \mathbf{a}, 3 \mathbf{3}, \mathbf{6} \mathbf{a}, 7 \mathbf{a}, \mathbf{b}$, or methyl $\alpha$ L-fucoside (11) at $100 \mu \mathrm{M}$. DMSO in absence of compounds was used as control. (A) Quantification of biofilm biomass. Averages and standard deviations of biofilm formation from three independent replicates. Statistical significance was calculated using the students $t$ test. (B) Raw data of confocal fluorescence microscopy 3D images show one representative $z$-stack per condition.

insignificant. In contrast, all glycomimetics tested showed a significant reduction of biofilm formation with novel sulfonamide $C$-glycosides 7 as most potent compounds reaching approximately $80-90 \%$ of inhibition. Mannose analogs $2 \mathrm{a}$ and $3 \mathrm{a}$ also inhibited with a reduced potency the formation of biofilms with 54 and $66 \%$ observed reduction, respectively.

Compound Selectivity for LecB over Host Lectins. Carbohydrate-binding proteins are ubiquitous and many play important roles in host innate immune defense. ${ }^{42}$ Thus, inhibitors of LecB carrying natural carbohydrate epitopes may also result in unspecific inhibition of human pathogenrecognition receptors. Langerin is a $C$-type lectin expressed in human Langerhans cells and $\mathrm{CD} 103^{+}$dermal dendritic cells and recognizes predominantly D-mannose but also L-fucose, $\mathrm{N}$ acetyl-D-glucosamine and sulfated carbohydrates. ${ }^{43,44}$ As the Cglycosides presented here contain structural elements of Dmannose, L-fucose, and sulfonamide moieties, they are potential ligands of Langerin. Therefore, this protein was chosen as one representative host lectin to study compound specificity. In order to assess the compounds' selectivity, 6a, 7a, and its natural ligand $\mathrm{L}$-fucose $(\mathbf{8})$ were analyzed for Langerin binding in a competitive ${ }^{19} \mathrm{~F} \mathrm{R}_{2}$-filtered NMR assay ${ }^{44}$ (Figure 7). Natural ligand $\mathbf{8}$ was recognized by Langerin with slightly decreased affinity ( $K_{i}$ of $\left.7.2 \mathrm{mM}\right)$ compared to D-mannose $\left(K_{i}\right.$ $\left.4.5 \mathrm{mM}^{44}\right) ; C$-glycosides $6 \mathrm{a}$ and $7 \mathrm{a}$ also showed moderate binding to this human protein in the millimolar range. However, both compounds displayed a 1000-fold stronger affinity for LecB and are therefore considered selective for LecB over the host lectin Langerin.

In addition to the direct binding to one host lectin, a more global effect of the glycomimetics on immune homeostasis was investigated. Primary murine spleen cells were stimulated via different pathways using bacterial lipopolysaccharide (LPS), fungal mannan, or a combination of phorbol 12-myristate 13acetate (PMA) and ionomycin, and the immune response to these stimuli in presence and absence of compounds $\mathbf{1}, \mathbf{2 a}, \mathbf{3 a}$, 6a, $7 \mathrm{a}$, or 11 was monitored by quantifying TNF- $\alpha$ release (Figure S6). No effect of mannoside 1 on the TNF- $\alpha$ levels was observed in comparison to matched DMSO concentrations (Figure S6A), while cinnamide 2a showed a decrease in TNF- $\alpha$ levels (Figure S6B). An immune suppressive effect was

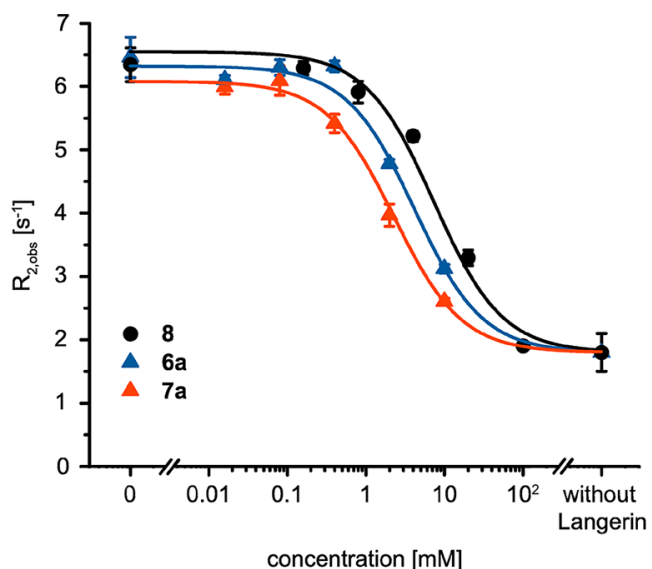

\begin{tabular}{llll} 
& $\mathbf{6 a}$ & $\mathbf{7 a}$ & $\mathbf{8}$ \\
\hline $\mathbf{K}_{\mathbf{i}}[\mathbf{m M}]$ & $4.0 \pm 0.1$ & $2.2 \pm 0.6$ & $7.2 \pm 2.2$ \\
\hline
\end{tabular}

Figure 7. Binding of $C$-glycosides $6 \mathbf{a}$ and $7 \mathbf{a}$ and $\mathrm{L}$-fucose (8) to Langerin as determined in a ${ }^{19} \mathrm{~F} \mathrm{R}_{2}$-filtered NMR competitive binding assay.

exclusively observed for cinnamide $\mathbf{2 a}$ at the highest concentration tested $(1 \mathrm{mM})$, while all other tested compounds, 3a, 6a, 7a, and 11, showed no effect on the immune response (Figure S6C). The immune-suppressive effect of $2 \mathrm{a}$ is an interesting observation; however, the high concentration needed probably impedes further development toward this effect. Excluding mannose-derived cinnamide 2a, none of the other compounds tested showed any effect on the immune response and provided further supporting evidence for compound selectivity.

In Vitro Metabolic Stability and Toxicity. To achieve effective compound concentrations in vivo, efficient compound uptake and stability toward host metabolism are crucial properties. Compound solubility is not considered problematic for these well-soluble carbohydrate derivatives. Calculated compound lipophilicity ( $\log \mathrm{P}$ ) showed reasonable values $(-1.25<\operatorname{cog} \mathrm{P}<-0.13)$ for all compounds selected for early pharmacokinetics and toxicity studies, i.e., sulfonamide $C$ glycosides $\mathbf{7 a}, \mathbf{b}$ derivatives and two mannose-based compounds $\mathbf{3 a}$ and $\mathbf{3 b}$ (Table S5).

In vitro metabolic stability of the LecB inhibitors was studied against mouse and human liver microsomes and murine plasma (Tables S6 and S7, Figure S7). The data obtained revealed a low intrinsic clearance $\left(\mathrm{CL}_{\text {int }}\right)$ by mouse and human liver microsomes for all tested compounds. The metabolic stability of the $C$-glycoside $7 \mathrm{a}\left(\mathrm{CL}_{\text {int }} 12 \mu \mathrm{L} / \mathrm{min} / \mathrm{mg}\right.$ protein $)$ toward mouse microsomes was slightly improved compared to its $O$ glycoside analog 3a $\left(\mathrm{CL}_{\text {int }} 19 \mu \mathrm{L} / \mathrm{min} / \mathrm{mg}\right.$ protein $)$ supporting the initial rational design of these $C$-glycosides. In the second group, the stabilities of mannoside $3 \mathbf{b}$ and $C$-glycoside $7 \mathbf{b}$ could not be differentiated since both compounds showed highest stability in this assay. Remarkably, all tested compounds showed very low clearance by human liver microsomes, where all substances fell into the highest stability category of this assay. Stability in murine plasma was assessed, and while the positive controls procaine and propoxycaine were rapidly degraded, all analyzed LecB ligands were as stable as the negative control procainamide and were not significantly degraded during the incubation period of $2 \mathrm{~h}$. Therefore, all 
compounds tested can also be considered stable in murine plasma.

In order to assess a first safety window, toxicity of compounds was studied in vitro using an immortalized human hepatocyte cell line (Hep G2 cells, Figure S8). No toxicity was detected for all compounds tested up to a concentration of $100 \mu \mathrm{M}$.

In Vivo Pharmacokinetics of C-Glycosides. The two candidates from sulfonamides 7 with potent antibiofilm effects and favorable ADME properties, i.e., $7 \mathbf{a}$ and $7 \mathbf{b}$, were chosen for an in vivo pharmacokinetics study in mice. Male CD-1 mice received a single dose of either $7 \mathbf{a}$ or $7 \mathbf{b}(10 \mathrm{mg} / \mathrm{kg} ; n=3)$ intravenously (i.v.) or perorally (p.o.). Plasma and urine levels of the parent compounds were monitored during $24 \mathrm{~h}$ (Figure 8) and pharmacokinetic parameters of $7 \mathbf{a}$ and $7 \mathbf{b}$ were assessed
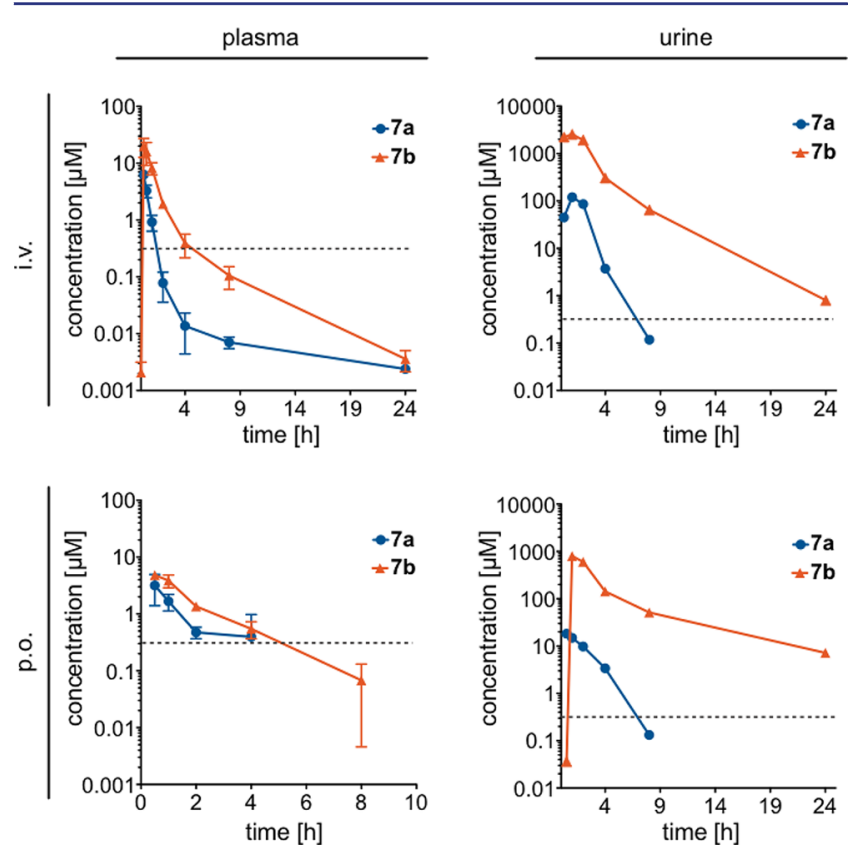

Figure 8. In vivo pharmacokinetics of $7 \mathbf{a}$ and $7 \mathbf{b}$ in CD-1 mice. Mean profile $( \pm)$ SD of plasma (left column) and urine (right column) concentration in mouse versus time after i.v. (top) or p.o. (bottom) administration of compound $7 \mathbf{a}$ or $7 \mathbf{b}$ in a single dosing experiment $(10 \mathrm{mg} / \mathrm{kg}, n=3)$. Dashed line represents the in vitro $\mathrm{IC}_{50}$ range for both candidates with LecB $\mathrm{PA14}_{\text {. }}$

from plasma levels using a one-compartment model (Table S10). ${ }^{45}$ In general, thiophene $7 \mathbf{b}$ had a superior plasma concentration over that of mesitylene $7 \mathbf{a}$ achieving a 2 -fold higher calculated initial concentration $\left(C_{0}=13.4 \mu \mathrm{M}\right.$ for $7 \mathbf{a}$ (i.v.); $C_{0}=28.3 \mu \mathrm{M}$ for $7 \mathbf{b}$ (i.v.)); thus, both candidates exceeded the anticipated necessary therapeutic levels (concentration $>$ target $K_{d}$ ) with $10 \mathrm{mg} / \mathrm{kg}$ i.v.. Furthermore, $7 \mathbf{b}$ showed a slower elimination rate $\left(k_{10}\right)$, a prolonged half-life $\left(t_{1 / 2}\right)$ and mean residence time (MRT) and a low clearance (CL) compared to 7a. This resulted in higher compound levels in plasma at later time points. These features also contribute to an overall drug exposure in plasma for $7 \mathbf{b}$ at an AUC of $7.4 \mu \mathrm{g}$. $\mathrm{h} / \mathrm{mL}$ using the intravenous administration route, which was around 4-fold higher than for $7 \mathbf{a}$. The p.o. administration of $7 \mathbf{a}$ resulted in a bioavailability of nearly $100 \%$, whereas for $7 \mathbf{b}$ it was only around $33.5 \%$. However, the p.o. route of administration of $\mathbf{7 b}$ still resulted in a higher AUC than that for $7 \mathbf{a}$ in the i.v. route. In addition, the p.o. route of $7 \mathbf{b}$ showed similar elimination curves as detected for the i.v. route. In general, both compounds $7 \mathbf{a}$ and $7 \mathbf{b}$ were absorbed rapidly ( $t_{\max }: 0.26-0.51 \mathrm{~h}$ for $7 \mathbf{a}$ and $7 \mathbf{b}$, p.o. route), which might be a consequence of the sugar-like structural elements inherited by both compounds facilitating the crossing of barriers. As unmetabolized $\mathbf{7 b}$ was detected in urine at high concentrations (Figure 8), its elimination from the systemic circulation likely proceeds via renal clearance rather than liver detoxification. The kidneys did not act as a reservoir, and thiophene $7 \mathbf{b}$ did not accumulate in this organ, since its concentration in kidney $24 \mathrm{~h}$ post i.v. dosing was below $6 \mathrm{nM}$. Taken together, both compounds showed good oral bioavailability and thiophene $7 \mathbf{b}$ showed a superior pharmacokinetic profile compared to $7 \mathbf{a}$ with high compound levels, both in plasma and urine.

\section{CONCLUSIONS}

We have developed the first low molecular weight LecB inhibitors that potently inhibited biofilm formation by $P$. aeruginosa, one of the prime mechanisms of antimicrobial resistance of this pathogen of outstanding medical importance. The synthesized glycomimetic $C$-glycosides showed inhibitory affinities as low as nanomolar for LecB variants from two strains representative for the entire set of clinical isolates. ${ }^{20}$ Thus, it can be expected that the devised glycomimetics will be active against LecB-mediated biofilm formation in a broad spectrum of clinical isolates.

The compounds showed excellent biophysical properties with receptor residence times as high as $28 \mathrm{~min}$ and fully enthalpy driven binding to the target lectin, which are two important benchmarks for early drug development. In contrast to the ineffective natural carbohydrate ligands, our glycomimetics were potent inhibitors of biofilm formation without affecting bacterial viability. Thus, development of resistances toward these pure antibiofilm substances is likely to be reduced, in contrast to this well-known problem for traditional antibiotics. $^{12,46}$

Target selectivity was then studied in binding experiments toward Langerin, a human fucose- and mannose-binding C-type lectin, as well as in a more global approach in immunestimulated primary murine spleen cells, and no impeding effects of the glycomimetics could be observed. All compounds tested showed absence of toxicity. Furthermore, a set of in vitro ADME experiments revealed good metabolic stability of the compounds against liver microsomes and murine and human plasma as well as a balanced lipophilicity that is important for oral availability.

In a first in vivo experiment, the pharmacokinetics of $7 \mathbf{a}$ and $7 \mathbf{b}$ in mice revealed oral bioavailability with high plasma concentrations and a subsequent primary excretion route via the kidneys. Consequently, high compound concentrations in urine were observed, suggesting but not limiting to an application in a $P$. aeruginosa urinary tract infection (UTI) model. It will now be of interest to evaluate the compounds' anti-infective potential in a monotherapy treatment against biofilm-associated infections and their synergy together with antibiotics for efficient eradication of bacteria outside the biofilm.

\section{ASSOCIATED CONTENT}

\section{Supporting Information}

The Supporting Information is available free of charge on the ACS Publications website at DOI: 10.1021/jacs.7b11133. 
Experimental details and ${ }^{1} \mathrm{H}$ and ${ }^{13} \mathrm{C}$ spectra of new compounds; ITC titration data; X-ray data collection and refinement statistics; crystal structures showing ligand alignment and crystal packing effects; correlation of fluorescence intensities with cfu and OD600 measurements; effects of compounds on total fluorescence intensities; calculated lipophilicity of selected compounds; analysis of TNF- $\alpha$ concentration after stimulation of mouse spleen cells with and without test compounds; microsomal intrinsic clearance $\left(\mathrm{CL}_{\mathrm{int}}\right)$ of $\mathbf{3 a}$, $3 \mathbf{b}$, and $C$-glycosides 7 in mouse and human liver microsomes; stability of LecB ligands in mouse plasma; $\mathrm{m} / \mathrm{z}$ search window for plasma stability assay; toxicity of LecB ligands to human liver Hep G2 cells; accuracy, quantification limits, and lower limit of qualification for $7 \mathbf{a}$ and $7 \mathbf{b}$ in plasma, urine, and kidney matrix; mass spectrometric conditions used for quantification and qualification of $7 \mathbf{a}, 7 \mathbf{b}$, and the internal standard glipizide; PK parameters of $7 \mathbf{a}$ and $7 \mathbf{b}$ in mice (PDF)

\section{AUTHOR INFORMATION}

\section{Corresponding Author}

*Tel. +49 68198806 2500. E-mail: alexander.titz@helmholtzhzi.de.

\section{ORCID}

Christoph Rademacher: 0000-0001-7082-7239

Rolf W. Hartmann: 0000-0002-5871-5231

Alexander Titz: 0000-0001-7408-5084

\section{Notes}

The authors declare no competing financial interest.

\section{ACKNOWLEDGMENTS}

We are grateful to Sarah Henrikus and Shelby Newsad for chemistry support and to Dr. Michael Hoffmann for HRMS measurements (all HIPS Saarbrücken). We acknowledge technical assistance from Tatjana Arnold (HZI, Braunschweig) and Astrid Glöckner (Konstanz University); Dr. Aymeric Audfray (CERMAV Grenoble) is acknowledged for instructions to SPR. We thank Dr. Josef Zapp (Saarland University) for performing NMR measurements. Crystal data collection was performed at the European Synchrotron Radiation Facility, Grenoble, France, and we are grateful for access and technical support to beamline FIP-BM30A and ID29. A.I. and A.V. acknowledge support from the ANR projects Glyco@Alps (ANR-15-IDEX-02) and Labex ARCANE (ANR-11-LABX003). We thank the Helmholtz Association (grant no VH-NG934, to A.T.), EU COST action BM1003 (to R.S.), DAAD RISE program (to S. Newsad and R.S.) and the Deutsche Forschungsgemeinschaft (to A.T., grant no. Ti756/2-1, to C.R., grant no. RA1944/2-1) for financial support.

\section{REFERENCES}

(1) Nagao, M.; Iinuma, Y.; Igawa, J.; Saito, T.; Yamashita, K.; Kondo, T.; Matsushima, A.; Takakura, S.; Takaori-Kondo, A.; Ichiyama, S. J. Hosp Infect 2011, 79, 49-53.

(2) Rice, L. B. J. Infect. Dis. 2008, 197, 1079-1081.

(3) Tsutsui, A.; Suzuki, S.; Yamane, K.; Matsui, M.; Konda, T.; Marui, E.; Takahashi, K.; Arakawa, Y. J. Hosp. Infect. 2011, 78, 317-322.

(4) World Health Organization. WHO publishes list of bacteria for which new antibiotics are urgently needed. http://www.who.int/ mediacentre/news/releases/2017/bacteria-antibiotics-needed/en/ (accessed May 2017).
(5) Hauser, A. R., Rello, J., Eds. Severe Infections Caused by Pseudomonas aeruginosa; Perspectives on Critical Care Infectious Diseases series; Kluwer Academic Publishers Group: Boston, MA, 2003.

(6) Serra, R.; Grande, R.; Butrico, L.; Rossi, A.; Settimio, U. F.; Caroleo, B.; Amato, B.; Gallelli, L.; de Franciscis, S. Expert Rev. AntiInfect. Ther. 2015, 13, 605-613.

(7) Mittal, R.; Aggarwal, S.; Sharma, S.; Chhibber, S.; Harjai, K. J. Infect. Public Health 2009, 2, 101-11.

(8) Poole, K. Front. Microbiol. 2011, 2, 65.

(9) Flemming, H.-C.; Wingender, J. Nat. Rev. Microbiol. 2010, 8, 623-633.

(10) Davies, D. Nat. Rev. Drug Discovery 2003, 2, 114-122.

(11) Sommer, R.; Joachim, I.; Wagner, S.; Titz, A. Chimia 2013, 67, 286-290.

(12) Wagner, S.; Sommer, R.; Hinsberger, S.; Lu, C.; Hartmann, R. W.; Empting, M.; Titz, A. J. Med. Chem. 2016, 59, 5929-5969.

(13) Winzer, K.; Falconer, C.; Garber, N. C.; Diggle, S. P.; Camara, M.; Williams, P. J. Bacteriol. 2000, 182, 6401-6411.

(14) Diggle, S. P.; Stacey, R. E.; Dodd, C.; Cámara, M.; Williams, P.; Winzer, K. Environ. Microbiol. 2006, 8, 1095-1104.

(15) Tielker, D.; Hacker, S.; Loris, R.; Strathmann, M.; Wingender, J.; Wilhelm, S.; Rosenau, F.; Jaeger, K.-E. Microbiology 2005, 151, 1313-1323.

(16) Gilboa-Garber, N. Methods Enzymol. 1982, 83, 378-385.

(17) Klockgether, J.; Cramer, N.; Wiehlmann, L.; Davenport, C. F.; Tümmler, B. Front. Microbiol. 2011, 2, 150.

(18) Dötsch, A.; Schniederjans, M.; Khaledi, A.; Hornischer, K.; Schulz, S.; Bielecka, A.; Eckweiler, D.; Pohl, S.; Häussler, S. mBio 2015, 6, e00749-15.

(19) Boukerb, A. M.; Decor, A.; Ribun, S.; Tabaroni, R.; Rousset, A.; Commin, L.; Buff, S.; Doléans-Jordheim, A.; Vidal, S.; Varrot, A.; Imberty, A.; Cournoyer, B. Front. Microbiol. 2016, 7, 811.

(20) Sommer, R.; Wagner, S.; Varrot, A.; Nycholat, C. M.; Khaledi, A.; Haussler, S.; Paulson, J. C.; Imberty, A.; Titz, A. Chem. Sci. 2016, 7, 4990-5001.

(21) Boukerb, A. M.; Rousset, A.; Galanos, N.; Méar, J.-B.; Thepaut, M.; Grandjean, T.; Gillon, E.; Cecioni, S.; Abderrahmen, C.; Faure, K.; Redelberger, D.; Kipnis, E.; Dessein, R.; Havet, S.; Darblade, B.; Matthews, S. E.; de Bentzmann, S.; Guéry, B.; Cournoyer, B.; Imberty, A.; Vidal, S. J. Med. Chem. 2014, 57, 10275-10289.

(22) Chemani, C.; Imberty, A.; de Bentzmann, S.; Pierre, M.; Wimmerová, M.; Guery, B. P.; Faure, K. Infect. Immun. 2009, 77, 2065-2075.

(23) Cott, C.; Thuenauer, R; Landi, A.; Kühn, K.; Juillot, S.; Imberty, A.; Madl, J.; Eierhoff, T.; Römer, W. Biochim. Biophys. Acta, Mol. Cell Res. 2016, 1863, 1106-1118.

(24) Mitchell, E.; Houles, C.; Sudakevitz, D.; Wimmerova, M.; Gautier, C.; Pérez, S.; Wu, A. M.; Gilboa-Garber, N.; Imberty, A. Nat. Struct. Biol. 2002, 9, 918-921.

(25) Hauber, H.-P.; Schulz, M.; Pforte, A.; Mack, D.; Zabel, P.; Schumacher, U. Int. J. Med. Sci. 2008, 5, 371-376.

(26) Bucior, I.; Abbott, J.; Song, Y.; Matthay, M. A.; Engel, J. N. Am. J. Physiol Lung Cell Mol. Physiol 2013, 305, L352-363.

(27) Cecioni, S.; Imberty, A.; Vidal, S. Chem. Rev. 2015, 115, 525561.

(28) Bernardi, A.; Jiménez-Barbero, J.; Casnati, A.; De Castro, C.; Darbre, T.; Fieschi, F.; Finne, J.; Funken, H.; Jaeger, K.-E.; Lahmann, M.; Lindhorst, T. K.; Marradi, M.; Messner, P.; Molinaro, A.; Murphy, P. V.; Nativi, C.; Oscarson, S.; Penadés, S.; Peri, F.; Pieters, R. J.; Renaudet, O.; Reymond, J.-L.; Richichi, B.; Rojo, J.; Sansone, F.; Schäffer, C.; Turnbull, W. B.; Velasco-Torrijos, T.; Vidal, S.; Vincent, S.; Wennekes, T.; Zuilhof, H.; Imberty, A. Chem. Soc. Rev. 2013, 42, $4709-4727$.

(29) Johansson, E. M. V.; Crusz, S. A.; Kolomiets, E.; Buts, L.; Kadam, R. U.; Cacciarini, M.; Bartels, K.-M.; Diggle, S. P.; Cámara, M.; Williams, P.; Loris, R.; Nativi, C.; Rosenau, F.; Jaeger, K.-E.; Darbre, T.; Reymond, J.-L. Chem. Biol. 2008, 15, 1249-1257. 
(30) Hauck, D.; Joachim, I.; Frommeyer, B.; Varrot, A.; Philipp, B.; Möller, H. M.; Imberty, A.; Exner, T. E.; Titz, A. ACS Chem. Biol. 2013, 8, 1775-1784.

(31) Sommer, R.; Exner, T. E.; Titz, A. PLoS One 2014, 9, e112822.

(32) Sommer, R.; Hauck, D.; Varrot, A.; Wagner, S.; Audfray, A.; Prestel, A.; Möller, H. M.; Imberty, A.; Titz, A. ChemistryOpen 2015, 4, 756-767.

(33) Hofmann, A.; Sommer, R.; Hauck, D.; Stifel, J.; GöttkerSchnetmann, I.; Titz, A. Carbohydr. Res. 2015, 412, 34-42.

(34) Beshr, G.; Sommer, R.; Hauck, D.; Siebert, D. C. B.; Hofmann, A.; Imberty, A.; Titz, A. MedChemComm 2016, 7, 519-530.

(35) Sabin, C.; Mitchell, E. P.; Pokorná, M.; Gautier, C.; Utille, J.-P.; Wimmerová, M.; Imberty, A. FEBS Lett. 2006, 580, 982-987.

(36) Tarcsay, A.; Keseru, G. M. Drug Discovery Today 2015, 20, 8694.

(37) Hopkins, A. L.; Keseru, G. M.; Leeson, P. D.; Rees, D. C.; Reynolds, C. H. Nat. Rev. Drug Discovery 2014, 13, 105-121.

(38) Tummino, P. J.; Copeland, R. A. Biochemistry 2008, 47, 54815492.

(39) Perret, S.; Sabin, C.; Dumon, C.; Pokorná, M.; Gautier, C.; Galanina, O.; Ilia, S.; Bovin, N.; Nicaise, M.; Desmadril, M.; GilboaGarber, N.; Wimmerová, M.; Mitchell, E. P.; Imberty, A. Biochem. J. 2005, 389, 325-332.

(40) Loris, R.; Tielker, D.; Jaeger, K.-E.; Wyns, L. J. Mol. Biol. 2003, 331, 861-870.

(41) Lagendijk, E. L.; Validov, S.; Lamers, G. E. M.; de Weert, S.; Bloemberg, G. V. FEMS Microbiol. Lett. 2010, 305, 81-90.

(42) Mayer, S.; Raulf, M.-K.; Lepenies, B. Histochem. Cell Biol. 2017, $147,223-237$.

(43) Holla, A.; Skerra, A. Protein Eng., Des. Sel. 2011, 24, 659-669.

(44) Wamhoff, E.-C.; Hanske, J.; Schnirch, L.; Aretz, J.; Grube, M.; Varón Silva, D.; Rademacher, C. ACS Chem. Biol. 2016, 11, 24072413.

(45) Zhang, Y.; Huo, M.; Zhou, J.; Xie, S. Comput. Methods Programs Biomed 2010, 99, 306-314.

(46) Allen, R. C.; Popat, R.; Diggle, S. P.; Brown, S. P. Nat. Rev. Microbiol. 2014, 12, 300-308.

\section{NOTE ADDED AFTER ASAP PUBLICATION}

This paper was published January 22, 2018. In the article and supporting information, compound $2 \mathrm{a}$ related to the X-ray structure has been corrected to $3 \mathrm{a}$. The revised version was posted on February 21, 2018. 